\title{
A COMPARATIVE STUDY OF TYPHIDOT TEST AND WIDAL TEST WITH THE CULTURE-POSITIVE TYPHOID FEVER IN CHILDREN
}

\author{
${ }^{1}$ Associate Professor, Department of Paediatrics, Chengalpattu Medical College. \\ ${ }^{2}$ Resident, Department of Paediatrics, ICH Egmore, Chennai. \\ 3 Professor, Department of Paediatrics, Chengalpattu Medical College. \\ ${ }^{4}$ Associate Professor, Department of Paediatrics, Stanley Medical College.
}

Jayakumar Muthaiyan1, Mohamed Rebois Abdul Moomin², Poovazhai Varadarajan³, Ganesh Jayachandran 4

\section{ABSTRACT}

\section{BACKGROUND}

Typhoid fever in children is a common childhood infection in both urban and rural regions in tropical countries. Clinical presentation is varied and overlaps many other acute febrile illness.

\section{MATERIALS AND METHODS}

Study was a prospective descriptive study. The study was conducted in Institute of Child Health and Hospital for Children, Egmore, Chennai, Tamilnadu during the period of April 2013 to November 2015. Children aged 3-12 years with fever more than 5 days with no obvious focus of infection. Coated tongue, abdominal distension, toxic look, constipation or loose stools, hepatomegaly, splenomegaly, relative bradycardia was included. Children immunised with typhoid vaccine, children already on antibiotics, and children with documented typhoid fever in the past 8 weeks were excluded. It was aimed to analyse the clinical presentation and to compare the Typhidot test and Widal test with the culture positivity in children with typhoid fever.

\section{RESULTS}

167 children fitted into the inclusion criteria, of which $26(15.6 \%)$ children were culture positive. 52 (31.1\%) children were Widal positive, $49(29.3 \%)$ were Typhidot test positive. Fever with anorexia was the common symptoms, and toxic look, coated tongue was the common signs in our study group.

\section{CONCLUSIONS}

Blood culture for typhoid fever is the gold standard, but is time consuming. In our study, we conclude that Typhidot shows high sensitivity and low specificity with higher negative predictive value when compared to Widal test.

\section{KEYWORDS}

Typhoid fever, Typhidot Test, Widal Test.

HOW TO CITE THIS ARTICLE: Muthaiyan J, Moomin MRA, Varadarajan P, et al. A comparative study of Typhidot test and Widal test with the culture-positive typhoid fever in children. J. Evolution Med. Dent. Sci. 2016;5(87):6476-6479, D0I: $10.14260 /$ jemds/2016/1464

\section{BACKGROUND}

Early and prompt diagnosis of typhoid is mandatory among children. Since the clinical features of the common infections like malaria, dengue, typhoid, leptospirosis and scrub typhus are very similar it is difficult for the treating physician to diagnose typhoid fever based on clinical features. $(1,2,3)$ It is then mandatory to have a very valid diagnostic test with good sensitivity and specificity for an earlier diagnosis in children with acute febrile illness. ${ }^{(4,5)}$ With increasing use of antibiotics, the clinical presentation is nonspecific and difficult to diagnose based on clinical features in children. The available blood culture for typhoid is time consuming and is affected by the prior antibiotic use. Conventional Widal test needs documentation of increasing titers and this is not useful for early therapy. Existing literatures on Typhidot test have

Financial or Other, Competing Interest: None.

Submission 23-09-2016, Peer Review 15-10-2016,

Acceptance 24-10-2016, Published 28-10-2016.

Corresponding Author:

Dr. Jayakumar Muthaiyan,

No. 5/4, $3^{\text {rd }}$ Cross Street,

Balaji Nagar, Ekkaduthangal,

Chennai-32.

E-mail:mjkumar67@rediffmail.com

DOI: $10.14260 /$ jemds $/ 2016 / 1464$ shown varied sensitivity and specificity among children and adults. $(6,7)$

\section{MATERIAL AND METHODS}

Aim

To study the clinical profile of typhoid fever in the paediatric age group and to compare the Typhidot test and Widal test with the culture positivity in children with typhoid fever.

\section{Study Design}

Prospective descriptive study. Setting: Institute of Child Health and Hospital for Children, Egmore, Chennai. Study period: April 2013 to November 2015. Study population: Children aged 3-12 years with Fever more than 5 days with no obvious focus of infection, coated tongue, abdominal distension, toxic look, constipation or loose stools, hepatomegaly, splenomegaly, relative bradycardia was included. Exclusion criteria: Children immunised with typhoid vaccine, children already on antibiotics, and children with documented typhoid fever in the past 8 weeks were excluded. Sample size: All children admitted in hospital who met the inclusion and exclusion criteria during the study period.

All children recruited for the study were subjected to a thorough clinical examination. Complete blood counts, peripheral smear, chest x-ray, liver function tests, Mantoux test, blood Widal after D7, urine culture and sensitivity, blood 
for enteric culture and Typhidot on day 5 of illness were done along with other investigations for fever evaluation as per unit protocol. Empiric treatment with Inj. Ceftriaxone was started. All children were followed up till defervescence of fever. They were observed for complications, response to treatment and alternative diagnosis.

Blood culture positivity is the gold standard for the diagnosis of typhoid fever. However, in view of need of early diagnosis, the serological test in the form of Typhidot can be a useful test bedside as the results are available within few minutes. The sensitivity and specificity though varied in different studies, overall this can be a useful test for the clinician to decide earlier therapy. Conventionally a positive Widal test implies increasing titers and this is not useful to decide therapy as it would need 2 weeks for the repeat sampling. The prior exposure of antibiotics will prevent the rising titres seen in truly affected children with typhoid fever. Hence, the role of these serological tests like Typhoid test in the clinical setting to decide on early therapy. Typhidot is a dot enzyme immunoassay that detects IgG and IgM antibodies against a $50 \mathrm{kD}$ outer membrane protein. This is distinct from the somatic (O), flagella $(\mathrm{H})$ or capsular (Vi) antigen of Salmonella typhi. Based on the existing literature this is an ideal bedside test for diagnosis of typhoid fever. Presence of IgM positivity indicates early phase of infection, presence of both IgM and IgG suggest middle phase of infection. IgG antibodies persist into 2 years of infection and this may not be of much use in acute infections. For this reason, the original Typhidot was modified as Typhidot $\mathrm{M}$ where the IgG levels will be masked and the results indicate the IgM values only and this is more sensitive and specific.

Descriptive data analysis was done. Study was undertaken after ethical clearance and informed written consent of the caregivers of these children. Statistical analysis was done using chi-square test for sensitivity, specificity, positive predictive value and negative predictive value for Widal test and typhoid test with culture positivity for typhoid fever as the gold standard test.

\section{RESULTS}

The study group included 167 children, among whom 94 $(56.3 \%)$ were male and $73(43.7 \%)$ were female children. 66 $(40 \%)$ of these children were in the 3-5 years age group, 64 (38\%) in the 6-8 years age group and $37(22 \%)$ in the $9-12$ years age group. The study group was divided into nontyphoid illness, confirmed typhoid fever and probable typhoid fever. 115 among the study group was non-typhoid illness like UTI, dengue, pneumonia, rickettsial fever, acute viral hepatitis and others. Culture-positive children were taken as typhoid and only Widal positive were taken as probable typhoid in this study.

The clinical features have been summarised in table 1 . Fever defervescence occurred at a mean of 5.8 days and of the 26 children, 25 had defervescence of fever by $8^{\text {th }}$ day. Laboratory features have been summarised in Table 2 .

26 were confirmed typhoid fever (Culture positive) and 52 were probable typhoid fever (Widal positive but culture negative). Of the 167 children, $15.6 \%$ i.e. 26 children were culture positive, 49 were Typhidot positive.

Among the culture positive 26 children, 21 were positive for Widal test. $\mathrm{O}$ was positive at 160 in 13 , up to 320 in 8 . $\mathrm{H}$ was positive in 160 in 12 and was positive in 320 in 8 children. Typhidot was positive in $23(88.5 \%)$ and 3 were negative.

Overall in the study group, 26 children were culture positive and 23 were positive in Typhidot. Among the 49 children with positive Typhidot test, 23 were culture positive and 26 were culture negative. Sensitivity of Typhidot was 88.5\% (CI 69.82-97.42), specificity was $81.56 \%$ (74.16-87.58), positive predictive value was $46.94 \%$ (32.54-61.720 and negative predictive value was $97.46 \%$ (92.74-99.44). Positive likelihood ratio was 4.8 (3.3-6.97). Negative likelihood ratio was 0.24 (0.05-0.41). Chi-square test was 51.916.

Analysis for Widal test revealed the following with the culture positivity. Of the 26 culture positive children, Widal was positive in 21 and 5 were negative. Overall, 52 children were positive for Widal test in the study group. Sensitivity of Widal test was 80.77 (60.64-93.37), specificity was 78.01 (70.22-84.54), positive predictive value was 40.38 (27.0154.9), negative predictive value was 95.65 (90.14-98.56) and chi square was 35.37. Positive likelihood ratio was 3.67 (2.565.28), negative likelihood ratio was 0.25 (0.11-0.54). None of the children in the study group developed any complications like pneumonia, ileus, encephalopathy or bleeds.

\begin{tabular}{|c|c|c|c|}
\hline Symptoms & $\begin{array}{c}\mathrm{N}=26 \\
(\%)\end{array}$ & Signs & $\begin{array}{c}\mathrm{N}-26 \\
(\%)\end{array}$ \\
\hline Fever & $\begin{array}{c}26 \\
(100)\end{array}$ & Toxic look & $\begin{array}{c}20 \\
(76.9)\end{array}$ \\
\hline Anorexia & $\begin{array}{c}17 \\
(65.4)\end{array}$ & Coated tongue & $\begin{array}{c}19 \\
(73.1)\end{array}$ \\
\hline Vomiting & $\begin{array}{c}17 \\
(65.4) \\
\end{array}$ & Hepatomegaly & $\begin{array}{c}16 \\
(61.5) \\
\end{array}$ \\
\hline Chills & $\begin{array}{c}16 \\
(61.5)\end{array}$ & Splenomegaly & $\begin{array}{c}12 \\
(46.2)\end{array}$ \\
\hline Cough & $\begin{array}{c}15 \\
(57.7)\end{array}$ & Hepatosplenomegaly & $\begin{array}{c}10 \\
(38.1)\end{array}$ \\
\hline $\begin{array}{l}\text { Abdominal } \\
\text { pain }\end{array}$ & $\begin{array}{c}12 \\
(46.2)\end{array}$ & Lymphadenopathy & $\begin{array}{c}7 \\
(26.9)\end{array}$ \\
\hline Headache & $\begin{array}{c}11 \\
(42.3)\end{array}$ & Pallor & $\begin{array}{c}5 \\
(38.4)\end{array}$ \\
\hline Diarrhoea & $\begin{array}{c}9 \\
(34.6)\end{array}$ & & \\
\hline $\begin{array}{l}\text { Abdominal } \\
\text { pain }\end{array}$ & $\begin{array}{c}3 \\
(11.5)\end{array}$ & & \\
\hline Constipation & $1(3.8)$ & & \\
\hline ALOC & $1(3.8)$ & & \\
\hline Epistaxis & $1(3.8)$ & & \\
\hline \multicolumn{4}{|c|}{ Table 1} \\
\hline
\end{tabular}

\begin{tabular}{|c|c|}
\hline Parameter & N-26 (\%) \\
\hline Haemoglobin<10 g\% & $14(53.8)$ \\
\hline WBC $<4,000$ cells/cu. mm & $3(11.5)$ \\
\hline WBC $4000-10,000$ cells/cu. mm & $12(46.5)$ \\
\hline WBC $>10,000$ cells/cu. mm & $11(42.3)$ \\
\hline Platelet $<1,00,000$ & $1(3.8)$ \\
\hline Elevated SGOT IU & $8(30.8)$ \\
\hline Elevated SGPT IU & $10(38.5)$ \\
\hline Table 2. Laboratory Findings among the Culture \\
Positive Children for Typhoid Fever
\end{tabular}

\section{DISCUSSION}

Typhoid fever is a common illness among children in tropical countries. Significant morbidity and mortality exist in children with typhoid disease. Earlier diagnosis and treatment are of paramount importance in children. Multidrug resistant strains 
have added to the problem in the recent past. Clinical presentation of these children has been the same with fever, toxic look, coated tongue, abdominal pain, lethargy and hepatosplenomegaly or both as shown in our study.(1,2,3) The commonly used Widal test is not useful in the first week of illness, an increase in titre of Widal needs to be present. The results may take up to 48 hours. Culture for typhoid is the gold standard and it has the advantage of identifying the antibiotic sensitivity too. This is $100 \%$ specific for the diagnosis of typhoid fever. However, the disadvantage is that the reports are available after a delay of 72 hours. Recent exposure to antibiotics adversely affect the results of culture sensitivity. Hence, the serological tests have evolved in the recent past like the Tubex and Typhidot test which identify the antibodies against typhoid fever and help in evaluation of children with typhoid aiding an earlier diagnosis. Typhidot test has been a useful test in the diagnosis of Typhoid fever in children and the results are available within 20 minutes in comparison to the other tests. In the context of diagnosis of typhoid fever in children, serological tests like Typhidot test have been found to be very useful. Typhidot test detects both IgM and IgG antibodies to a Salmonella typhi-specific outer membrane protein. It is easier and quicker to perform. In order to increase diagnostic accuracy in an area of high endemicity, the Typhidot-M test has been developed in which IgG is first removed. This test has been claimed to recognise recent infections in children with typhoid fever.

Literature has revealed varied results for the serological tests when compared to the gold standard test. The sensitivity, specificity, positive predictive value and negative predictive value of various other available studies have been discussed below. The results of these sensitivity varies from $70-100 \%$ in various studies as also is the specificity. In studies by Beig et al(4) blood culture was positive in only $27.3 \%$ of the cases. Among these culture positive cases, Typhidot $\mathrm{M}$ test had the highest sensitivity, specificity, PPV and NPV of 90\% (95\% CI = 74.4-96.5), $100 \%$ (95\% CI = 90.1-100), 100\% (95\% CI = 87.5100), and $92.1 \%$ (95\% CI $=79.2-97.3)$ respectively. ${ }^{(4)}$ The present study revealed a sensitivity of $88.6 \%$, specificity was $81.6 \%$, positive predictive value was $46.94 \%$ and negative predictive value was $97.46 \%$.

Studies from Malaysia(5) have shown that the Typhidot test is more sensitive and specific in comparison to Widal tests. (Sensitivity $=85-94 \%$ and specificity $=77-89 \%$ ) Studies from Papua, New Guinea(6) have shown the results not very promising to include these serological tests in routine evaluation in their setting. The sensitivity of Typhidot test was $70 \%$ and specificity was $80 \%$. Studies have shown the sensitivity and specificity to be much higher if the pre-test probability was high as $50 \%$ in comparison to $5 \%$. These tests may be useful in emergency settings like outbreaks. Studies from Vietnam(7) have suggested that the sensitivity and specificity was 79 and $89 \%$ for Typhidot based on their studies. Most of the assays like Typhidot, Widal tests, Tubex test were found to be more sensitive during the second week of illness. Regional variation in the Typhidot results may be due the differences in the genomic structure and plasticity of the OMP of S. typhi and for this reason further evaluation of these tests in diverse geographic locations is warranted.(5) Studies from Bangladesh(8) have not found the test results to be very sensitive or specific. The results were - Typhidot ${ }^{\circledR}$ was
$67 \%$ sensitive and $54 \%$ specific, with $85 \%$ positive and $81 \%$ NPVs.

Studies from India ${ }^{(9)}$ revealed the following results. 105 children with clinical diagnosis of typhoid fever were evaluated with culture, Widal and Typhidot M. Of the 105 cases, blood culture was positive for S. typhi in 41 (39\%) children. In children with fever of less than 7 days duration, Typhidot-M was positive in $97 \%$, compared to $24.2 \%$ by Widal test. Typhidot-M was reported to be a simple and sensitive test for early diagnosis of typhoid fever in children. Studies from Bangalore,(10) India have shown the sensitivity to be $100 \%$. The sensitivity, specificity, positive and negative predictive values of the test using blood culture as gold standard were $100 \%, 95.5 \%, 89.2 \%$, and $100 \%$, respectively for typhoid fever. Studies from 100 children in Rajasthan have identified $20 \%$ culture positive, and the sensitivity, specificity, positive predictive value and negative predictive values were 95\%, $100 \%, 100 \%$ and $93 \%$ respectively. Recently published data from Chennai(11) has revealed Typhidot to be a very sensitive test in children with typhoid fever. Typhidot positivity was seen at a mean of 5.5 days of illness. The day of illness into the sampling did not significantly affect the results. Their sensitivity was $96.43 \%$, specificity was $54.4 \%$, positive predictive value was $25.96 \%$, negative predictive value was 98.92\% among the 197 children.

\section{CONCLUSIONS}

Fever with toxic look, anorexia, vomiting, coated tongue, hepatosplenomegaly are the common features of children with typhoid disease in this study.

Laboratory findings of complete blood count and liver enzymes were not contributory for the diagnosis.

Typhidot was found to have a sensitivity, specificity, positive predictive value and negative predictive value of $96.43 \%, 54.4 \%, 25.96 \%$ and $98.92 \%$ respectively.

\section{Recommendations}

Compared to Widal test, Typhidot can be done in the earlier phase of illness and the results are available within 30 minutes to decide on therapy. The high negative predictive value may be useful to avoid unnecessary antibiotic exposure in children with acute febrile illness.

Based on this study, Typhidot can be used as a simple, easy, reliable diagnostic test for children with an acute febrile illness where typhoid is suspected. Blood culture is the gold standard for diagnosis and to identify the antibiotic sensitivity, but Typhidot is a useful test to start an earlier specific therapy.

\section{ACKNOWLEDGEMENT}

The authors would like to acknowledge children, care givers and hospital staff, and Mr. M. Janaki Raman (System Analyst) for their kind cooperation.

\section{REFERENCES}

1. Malik AS, Malik RH. Typhoid fever in Malaysian children. Med J Malaysia 2001;56(4):478-90.

2. Khan NM, Shafee M, Hussain K, et al. Typhoid fever in paediatric patients in Quetta, Balochistan, Pakistan. Pak J Med Sci 2013;29(4):929-32.

3. Siddiqui FJ, Rabbani F, Hasan R, et al. Typhoid fever in children: some epidemiological considerations from Karachi, Pakistan. Int J Infect Dis 2006;10(3):215-22. 
4. Beig FK, Ahmad F, Ekram M, et al. Typhidot M and Diazo test vis-à-vis blood culture and Widal test in the early diagnosis of typhoid fever in children in a resource poor setting. Braz J Infect Dis 2010;14(6):589-93.

5. Choo KE, Davis TM, Ismail A, et al. Rapid and reliable serological diagnosis of enteric fever: comparative sensitivity and specificity of Typhidot and Typhidot-M tests in febrile Malaysian children. Acta Trop 1999;72(2):175-83.

6. Keddy KH, Sooka A, Letsoalo ME, et al. Sensitivity and specificity of typhoid fever rapid antibody tests for laboratory diagnosis at two sub-Saharan African sites. Bull World Health Organ 2011;89(9):640-7.

7. Olsen SJ, Pruckler J, Bibb W, et al. Evaluation of rapid diagnostic tests for typhoid fever. J Clin Microbiol 2004; 42(5):1885-9.
8. Naheed A, Ram PK, Brooks WA, et al. Clinical value of Tubex and Typhidot rapid diagnostic tests for typhoid fever in an urban community clinic in Bangladesh. Diagn Microbiol Infect Dis 2008;61(4):381-6.

9. Narayanappa D, Sripathi R, Jagdishkumar K, et al. Comparative study of dot enzyme immunoassay (Typhidot-M) and Widal test in the diagnosis of typhoid fever. Indian Pediatr 2010;47(4):331-3.

10. Krishna S, Desai S, Anjana VK, et al. Typhidot (IgM) as a reliable and rapid diagnostic test for typhoid fever. Ann Trop Med Public Health 2011;4(1):42-4.

11. Kumar KS, Suganya M, Sathyamurthi B, et al. Reliability of Typhidot Rapid Immunoglobulin M and Immunoglobulin $\mathrm{G}$ in the diagnosis of Typhoid fever. International $\mathrm{J}$ of Scientific Study 2016;4(2):256-9. 